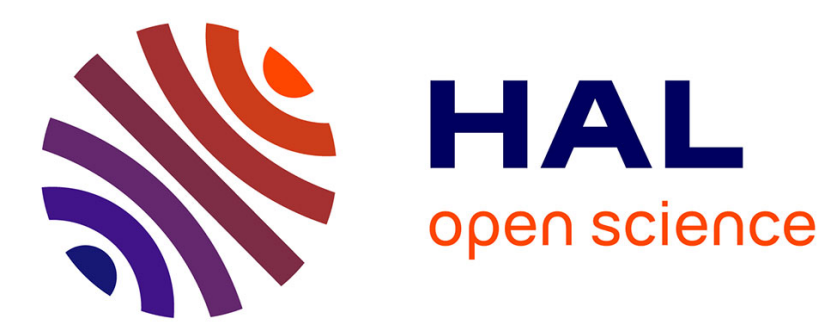

\title{
Converging approximations for the calculation of the energy in terms of Planck's constant
}

\author{
B.G. Giraud
}

\section{To cite this version:}

B.G. Giraud. Converging approximations for the calculation of the energy in terms of Planck's constant. Journal de Physique Lettres, 1979, 40 (2), pp.15-17. 10.1051/jphyslet:0197900400201500 . jpa-00231558

\section{HAL Id: jpa-00231558 https://hal.science/jpa-00231558}

Submitted on 1 Jan 1979

HAL is a multi-disciplinary open access archive for the deposit and dissemination of scientific research documents, whether they are published or not. The documents may come from teaching and research institutions in France or abroad, or from public or private research centers.
L'archive ouverte pluridisciplinaire HAL, est destinée au dépôt et à la diffusion de documents scientifiques de niveau recherche, publiés ou non, émanant des établissements d'enseignement et de recherche français ou étrangers, des laboratoires publics ou privés. 


\title{
LE JOURNAL DE PHYSIQUE-LETTRES
}

Classification

Physics Abstracts

$03.65-21.10 \mathrm{D}-21.60-31.20 \mathrm{D}$

\section{Converging approximations for the calculation of the energy in terms of Planck's constant $(*)$}

\author{
B. G. Giraud \\ Division de la Physique, Service de Physique Théorique, \\ CEN-Saclay, B.P. no 2, 91190 Gif sur Yvette, France.
}

(Reçu le 2 novembre 1978, accepté le 29 novembre 1978)

\begin{abstract}
Résumé. - Afin de calculer l'énergie quand $\hbar$ est petit, on développe le second membre de l'équation de BrillouinWigner en fonction d'une constante de couplage $\lambda$. Les hiérarchies en $\hbar$ et $\lambda$ se correspondent. Les approximants de Padé du développement du second membre convergent.
\end{abstract}

\begin{abstract}
In order to calculate the energy when $\hbar$ is small, we expand the right-hand side of the BrillouinWigner equation in terms of a coupling constant $\lambda$. Both hierarchies in $\hbar$ and $\lambda$ are connected. The Padé approximants of the right-hand side converge.
\end{abstract}

The present letter intends to show how the BrillouinWigner perturbation theory, combined with Padé approximants, can provide the calculation of eigenenergies of a quantum system with a converging and regular algorithm when Planck's constant vanishes.

For this purpose the unperturbed Hamiltonian $\mathscr{H}_{0}$ of the system under study is chosen as that of a harmonic oscillator. The unperturbed ground state and energy are labelled $\Phi_{0}$ and $\varepsilon_{0}$, respectively. The total Hamiltonian $\mathscr{H}$ is split into $\mathscr{H}_{0}$ and a perturbation $\lambda V$, with $\lambda=1$ in the physical case. The true eigenenergies $E_{n}$ of the system are the solutions of the BrillouinWigner equation

$$
\begin{gathered}
E(\lambda)=\varepsilon_{0}+F(E, \lambda), \quad \lambda=1 \\
F(E, \lambda) \equiv\left\langle\Phi_{0}\left|\lambda V+\lambda V \frac{Q}{E-\mathcal{H}_{0}-\lambda Q V Q} \lambda V\right| \Phi_{0}\right\rangle \\
=\sum_{k=1}^{\infty} \lambda^{k}\left\langle\Phi_{0}\left|V\left(\frac{Q}{E-\mathscr{H}_{0}} V\right)^{k-1}\right| \Phi_{0}\right\rangle
\end{gathered}
$$

where $Q \equiv 1-\left|\Phi_{0}\right\rangle\left\langle\Phi_{0}\right|$. Let $M$ be a positive integer. By means of those terms of degree lower or equal to $2 M$ of the perturbation expansion of $F$, eq. (2), with respect to $\lambda$, one can construct a Padé

(*) La traduction en français de cet article a été proposée au n ${ }^{\circ} 13$, série B des Comptes Rendus de l'Académie des Sciences du 13 novembre 1978. approximant $[M / M]$ of $F, F_{M}(E, \lambda)$. If one replaces eq. (1) by the approximate equation

$$
E(\lambda)=\varepsilon_{0}+F_{M}(E, \lambda)
$$

the solutions $E_{n M}$ of this equation are approximations of the exact eigenvalues $E_{n}$.

When $V$ is positive semi-definite it can be shown [1] that the values $E_{n M}$ make an increasing sequence of lower bounds to $E_{n}$, which converge when $M \rightarrow \infty$. It is also trivial to show that they make a decreasing sequence of upper bounds when $V$ is negative semidefinite. In both cases the main part of the proof is the reconstruction by bounds [2, 3], of the generalized Stieltjes function $F$ by means of its Padé approximants with respect to $\lambda$. Regularization techniques [3] are available if necessary.

An interesting feature of the algorithm described by eq. (3) is the dependence of $F_{M}$ upon $\hbar$. As a matter of fact, it is often possible to choose the splitting of a given $H_{\text {into }} H_{0}$ and $V$ in such a way that the $k$ th order term of the perturbation expansion described by eq. (2) is regular when $\hbar \rightarrow 0$ and, not only contains $\lambda^{k}$, but also $\hbar^{k}$ as a factor. The algorithm thus appears to be, in that sense, a semi-classical algorithm for the calculation of $F$, or $F_{M}$, and finally of $E_{n}$, in terms of a hierarchy of powers of $\hbar$.

This assertion can be first proved, for an illustrative example, in the case of a one-dimensional harmonic 
oscillator. Let $\mu$ be its mass and $\omega$ its frequency. One chooses

$\mathscr{H}_{0}=-\frac{\hbar^{2}}{2 \mu} \frac{\mathrm{d}^{2}}{\mathrm{~d} r^{2}}+\frac{1}{2} \mu \omega_{0}^{2} r^{2}$, with $\omega_{0}>\omega$

and thus the perturbation

$$
V=\frac{1}{2} \mu\left(\omega^{2}-\omega_{0}^{2}\right) r^{2}
$$

is negative semi-definite. It is then trivial to check that any matrix element of $V$ between eigenstates of $\mathcal{H}_{0}$ brings in a factor $\hbar$. The presence of $\hbar$ in the energy denominators due to $Q /\left(E-\mathfrak{H}_{0}\right)$ does not change the global factor $\hbar^{k}$ which appears in the $k$ th order term of the expansion, eq. (2).

A graphical solution of eq. (3) for the calculation of the ground state energy $E_{0}$ is given by figure 1 in the case where $\omega_{0}=2 \omega$, with $M=1$ and $M=2$. One finds, as expected, a decreasing sequence $\varepsilon_{0}=\hbar \omega$, $E_{01}=0.549 \hbar \omega, E_{02}=0.507 \hbar \omega$, in fast convergence towards $E_{0}=1 / 2 \hbar \omega$.

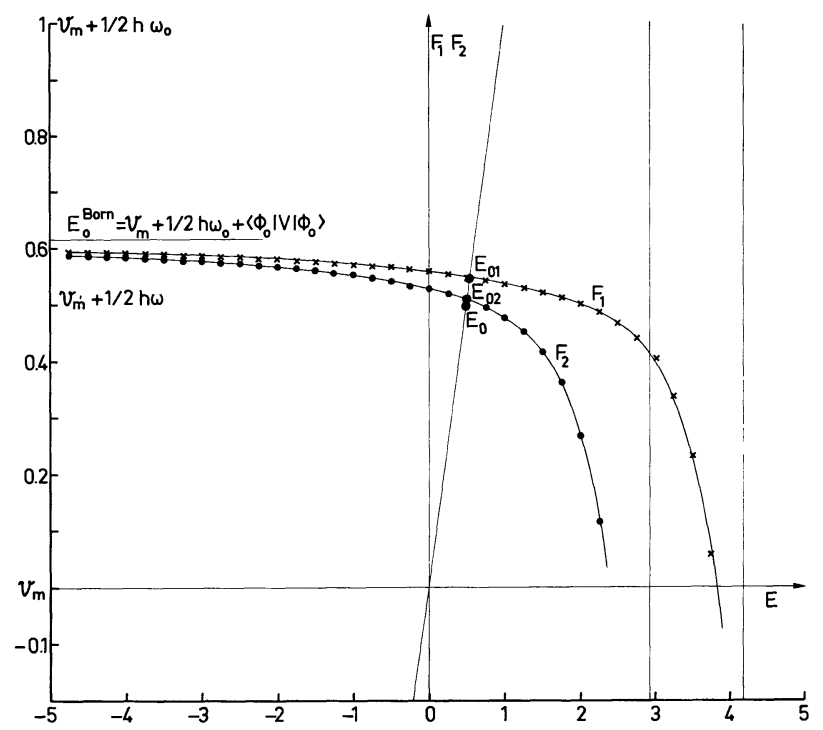

Fig. 1.

In the more general case of a one-dimensional particle with a local non-harmonic potential $V(r)$ one assumes that $\vartheta$ reaches an absolute minimum $\mho_{m}$ at $r_{\mathrm{m}}$ and that its Taylor series around $r_{\mathrm{m}}$ converges. By means of the second derivative $\vartheta_{m}^{\prime \prime}$ of $\vartheta$ at $r_{m}$ one defines the elementary length $l=\left[\hbar^{2} /\left(\mu \vartheta_{\mathrm{m}}^{\prime \prime}\right)\right]^{1 / 4}$ and a scaling length $l_{0}=\alpha l$ with $0<\alpha \leqslant 1$. The parameter $\alpha$ will remain temporarily fixed. One defines a scaling energy $e_{0}=\hbar^{2} /\left(\mu l_{0}^{2}\right)$. The shift and scaling operations $r=r_{\mathrm{m}}+l_{0} \rho$ and $E=\mathcal{V}_{\mathrm{m}}+e_{0} \varepsilon$ reduce the Schrödinger equation into

$$
\begin{aligned}
& {\left[-\frac{1}{2} \frac{\mathrm{d}^{2}}{\mathrm{~d} \rho^{2}}+\frac{1}{2} \alpha^{4} \rho^{2}+\frac{1}{6} \alpha^{5} \frac{\mathfrak{V}_{\mathrm{m}}^{\prime \prime \prime} l}{V_{\mathrm{m}}^{\prime \prime}} \rho^{3}+\right.} \\
& \left.\quad+\frac{1}{24} \alpha^{6} \frac{V_{\mathrm{m}}^{(4)} l^{2}}{V_{\mathrm{m}}^{\prime \prime}} \rho^{4}+\cdots-\varepsilon\right] \varphi(\rho)=0 .
\end{aligned}
$$

As well as the quantities $l, l_{0}$ and $e_{0}$, the dimensionless numbers $\vartheta_{\mathrm{m}}^{(p)} l^{p-2} / \vartheta_{\mathrm{m}}^{\prime \prime}$ derived from the derivatives of $\vartheta$ at $r_{\mathrm{m}}$ of order $p>2$ vanish when $\hbar \rightarrow 0$. For instance the coefficient of $\rho^{3}$ is of order $\hbar^{1 / 2}$. When multiplied by the scaling energy $e_{0}$, which is of order $\hbar$, the contribution of the corresponding matrix element to the calculation of $F$ is thus of order $\hbar^{3 / 2}$. More generally, any anharmonic term $V_{\mathrm{m}}^{(p)}\left(r-r_{\mathrm{m}}\right)^{p}$ has matrix elements going like $\hbar^{p / 2}$, thus of order $\hbar$ at least since $p>2$.

If therefore one chooses

$$
\begin{aligned}
H_{0} & =-\frac{\hbar^{2}}{2 \mu} \frac{\mathrm{d}^{2}}{\mathrm{~d} r^{2}}+\vartheta_{\mathrm{m}}+\frac{1}{2} \vartheta_{\mathrm{m}}^{\prime \prime} \alpha^{-4}\left(r-r_{\mathrm{m}}\right)^{2} \\
V & =\vartheta-\vartheta_{\mathrm{m}}-\frac{1}{2} \vartheta_{\mathrm{m}}^{\prime \prime} \alpha^{-4}\left(r-r_{\mathrm{m}}\right)^{2}
\end{aligned}
$$

the $k$ th order term with respect to $V$ in the perturbative calculation of $F$ does still contain a factor $\hbar^{k}$. This is however provided that the series of matrix elements induced by the Taylor series converges. The fast decay of harmonic oscillator wave functions when $|r| \rightarrow \infty$ should help [4] such a convergence in practical cases.

We need still to adjust $\alpha$ so that $V \leqslant 0$. If $\vartheta_{\mathrm{m}}^{\prime \prime \prime}=0$, which is not unusual in practical cases, one may take $\alpha=1$, with the benefit of matrix elements of $V$ vanishing like $\hbar^{2}$. If $\vartheta_{\mathrm{m}}^{\prime \prime \prime} \neq 0$, there are often (see Fig. 2) values of $\alpha<1$ for which $V \leqslant 0$, except when $\vartheta$ rises too strongly somewhere.

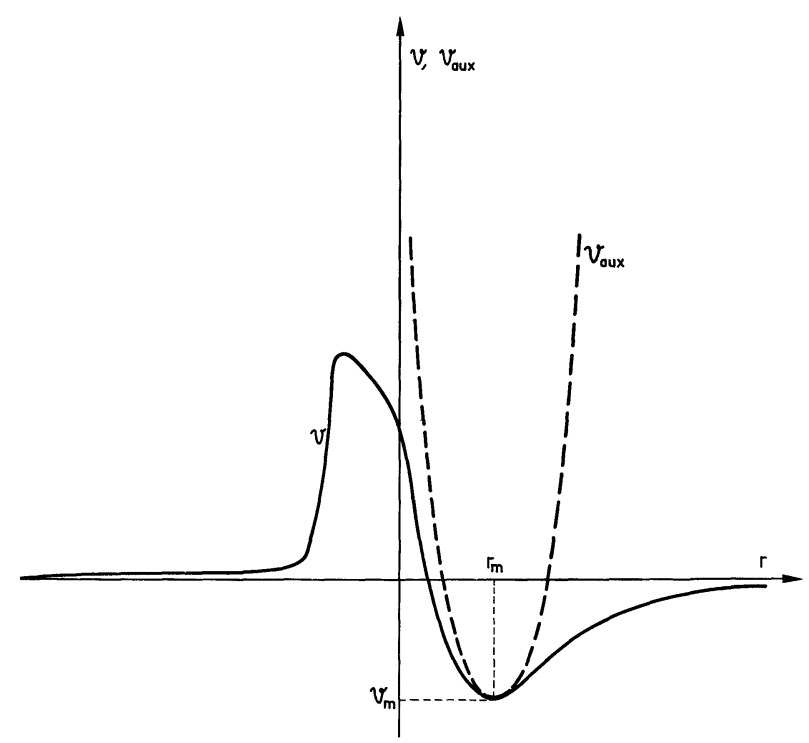

Fig. 2.

It is trivial to generalize these considerations to the case of $N$ particle in $d$ dimensions. One can use a hyperspherical, $N d$-dimensional formalism. This holds provided local $\vartheta$, which now depends on $N d$ coordinates, has a unique absolute minimum. Kinematical degeneracies (translations, rotations) of this minimum would induce the breaking of symmetries by the choice of $\mathcal{H}_{0}$. Symmetry restauration is possible, however [5]. 
In conclusion one may by-pass the singularities [6] of an expansion of the energy in terms of $\hbar$, whose nature in $\exp \{1 / \hbar\}$ has recently [7] been analysed, by an alternate expansion of the right-hand side of the Brillouin-Wigner equation. Convergence when $\hbar \rightarrow 0$ is obtained by a positivity condition and the use of Padé approximants.

This work has benefited from stimulating discussions with E. Brézin and H. Orland, to whom we would like to express our thanks.

\section{References}

[1] Giraud, B. G., Phys. Rev. C 17 (1978) 800. Turchetti, G., Ortolani, F. and Sagretti, C., Nuovo Cimento 44A (1978) 211.

[2] Bessis, D. and Villani, M., J. Math. Phys. 16 (1975) 462. BAKer, G., Essentials of Padé Approximants (Academic Press, N. Y.) 1975.

[3] Bessis, D., Epele, L. and Villani, M., J. Math. Phys. 15 (1974) 2071.

[4] Simon, B., Point wise bounds on eigenfunctions and wave-packets in N-body quantum systems, Trans. Ann. Phys. Soc., to be published.

[5] GiRaud, B., Restauration des Symétries brisées par les approxi- mations de Physique nucléaire, Thèse, Univ. de Paris XI (1972).

[6] Kato, T., Perturbation theory for linear operators (Springer Verlag, N. Y.) 1966 ;

MasLov, Théorie des perturbations et méthodes asymptotiques (Dunod) 1971;

CombeS, J. M., Asymptotic expansions for quantum mechanical bound-state energies near the classical limit, Proc. « Symposium on Spectral and Scattering theory", RIMS, Kyoto (1975).

[7] Balian, R., Parisi, G. and Voros, A., preprint Saclay DPh-T $78 / 20$. 